\title{
Stability of positive-position feedback controllers with low-frequency restrictions
}

\author{
Emiliano Pereira ${ }^{\mathrm{a}}$, Sumeet S. Aphale ${ }^{\mathrm{b}}$ \\ ${ }^{a}$ Escuela Politécnica Superior, Universidad de Alcalá, Ctra. Madrid-Barcelona, Km. \\ 33,600, 28805, Alcalá de Henares, Madrid (Spain) \\ ${ }^{b}$ Centre for Applied Dynamics Research, School of Engineering, Kings College, \\ University of Aberdeen, AB24 3UE, Aberdeen (UK)
}

\begin{abstract}
Positive-position feedback has been presented as a suitable alternative to the negative-velocity feedback approach taken in active damping applications. This has motivated the application of Negative-Imaginary Systems theory to study the stability of controllers based on positive-position feedback. However, this theory cannot be applied in cases where low-frequency dynamics of the sensors, actuators and/or controllers hinder the application of Negative-Imaginary stability conditions. This work extends the theory of Negative-Imaginary Systems by obtaining simple analytical stability conditions when such low-frequency restrictions are present.
\end{abstract}

Keywords:

positive position feedback, positive-real systems, vibration control, low-frequency restrictions, Negative-Imaginary Systems

Email address: Tel: 91-885-6711, Fax: 91-885-6889, Email: Emiliano.Pereira@uah.es (Emiliano Pereira) 


\section{Introduction}

Flexible structures are used in a variety of industrial, scientific as well as defence applications $[1,2,3,4]$. These structures are prone to vibrations caused by natural-frequency excitations which if left uncontrolled, may lead to unwanted displacements, positioning errors and in severe cases, failure due to fatigue. This has motivated a huge amount of research in the broad field of vibration control of flexible structures $[5,6,7,8]$. It is quite common to employ co-located sensor-actuator pairs in controlling flexible structures. Such structures are commonly referred to as co-located smart structures or co-located structures/systems. Such co-located systems exhibit the interesting property of pole-zero interlacing in the frequency-domain. Consequently, their phase response lies continuously between $0^{\circ}$ and $180^{\circ}$. Various vibration damping techniques exploiting this property have been proposed by researchers in the past. Velocity feedback achieves damping by using this property to implement a very simple derivative controller $[9,10]$. In practice however, velocity feedback presents some limitations. For instance, the requirement of a differentiator in piezoelectric laminated structures involves the inclusion of extra dynamics, which have the potential to destabilize the closed-loop system [11]. In addition, a high control effort at all frequency is required, thereby limiting the imparted damping at the desired frequencies. The resulting low performance and poor phase margins have motivated the Resonant controller, which has been applied successfully to highly resonant co-located systems $[12,13,14]$. A typical Resonant controller can be viewed as a negative velocity feedback controller in a narrow frequency band. This adjustment effectively reduces the control effort and increases the imparted 
damping at the desired frequency. However, as its response does not roll-off at higher frequencies, this control technique may not be suitable in certain applications where out-of-bandwidth noise is a critical issue. To alleviate this problem, Positive Position Feedback (PPF) has been proposed and experimentally demonstrated $[15,16,17]$. The main drawback of control techniques such as PPF and Resonant control is that they produce a second-order controller to damp a single resonant mode of the structure, thus resulting in a high-order controller for damping multiple modes. Also, they may be difficult to tune for cases where multiple modes need to be damped. This has further motivated the formulation of the Integral Resonant Control (IRC) [18], which combines the simplicity of a velocity feedback with the stability margins of a PPF.

Positive feedback controllers are more robust against uncertainty in the modal frequencies as well as unmodeled plant dynamics when compared with negative feedback controllers. This has motivated the application of Negative-Imaginary System (NIS) theory to study the stability of positive feedback controllers [19, 20]. NIS theory has been merged with small-gain theorems to establish the stability of interconnected systems [21]. It has been demonstrated here that in active damping applications, NIS theory can be applied in the bandwidth of interest (middle-frequency) by including a low-pass filter to roll-off the magnitude and achieve the small-gain theorems. In this case, the high frequency dynamics of the system are unmodeled and are not considered in the controller design. However, the effect of the low frequency dynamics introduced by sensor/s, actuator/s as well as the controller/s themselves on the overall stability margins, which hinder the 
application of NIS theory, has not been studied analytically. This work proposes simple analytical stability conditions when low-frequency restrictions are present due to sensors and/or actuators [22, 23]. An example of such a scenario is the implementation of the modified IRC technique required to reduce the control effort at low frequencies, as presented in [18].

\section{Preliminaries}

The control scheme shown in Fig. 1, which can be configured as PPF or IRC to reduce the vibrations caused by the perturbation $w$, is studied in this work. $G_{A}(s)$ is the transfer function $(\mathrm{TF})$ of the actuator, $G_{S}(s)$ is $\mathrm{TF}$ of the sensor, $G_{y u}(s)$ is the co-located TF between the output $(y)$ and input $(u), G_{y w}(s)$ is the TF between the output (y) and disturbance input $(w), D_{f}$ is a feed-through term such that $D \in \Re$ and is used to introduce a pair of complex conjugate zeros at a frequency lower than the system resonant poles. This results in a phase inversion at DC relative to the original co-located TF $G_{y u}(s)[18]$, and $C(s)$ is the TF of the controller.

\section{Stability proof}

The control scheme in Fig. 1 can be rearranged as Fig. 2. This simplifies the stability analysis for the system that now includes the co-located TF $G_{y u}(s)$ and the equivalent controller $\hat{C}(s)$. The stability of the control scheme of Fig. 2 can now be demonstrated by considering the positive feedback interconnection of $G_{y u}(s)$ and $\hat{C}(s)$. At this point, the results proved in [19] and [20], which are based on the feedback connection of systems with Negative- 


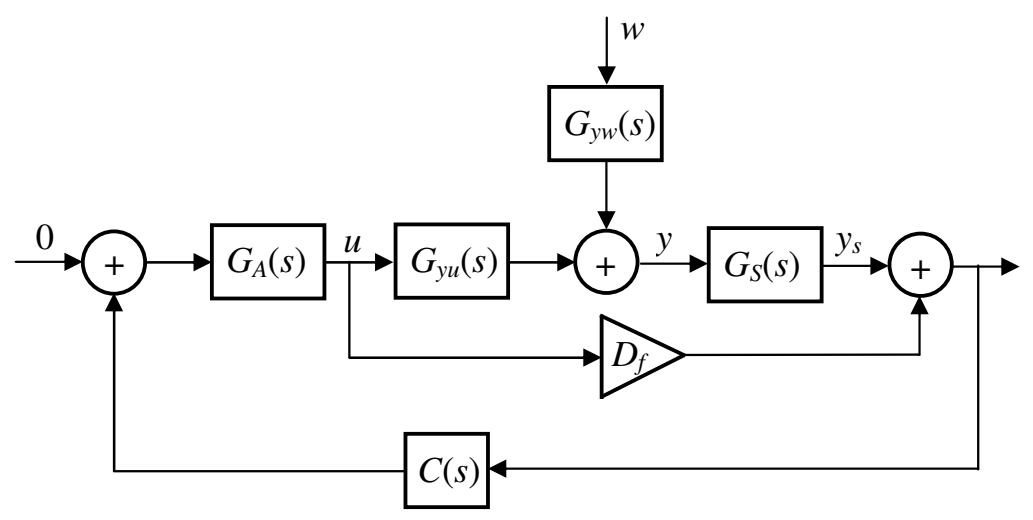

Figure 1: Positive-position feedback. General control scheme.

Imaginary Frequency Response, (NIFR) ${ }^{1}$ are utilized. More precisely, it is derived from the Theorem 5 of [19] that the positive feedback connection of two stable single-input single-output (SISO) systems $M(s)$ and $N(s)$, with $j\left[M(j \omega)-M(j \omega)^{*}\right] \geq 0$ and $j\left[N(j \omega)-N(j \omega)^{*}\right]>0 \quad \forall \omega \in(0, \infty)$, is internally stable if and only if $M(0) N(0)<1, M(\infty) N(\infty)=0$ and $N(\infty) \geq 0$.

The co-located transfer function, denoted by $G_{y u}(s)$, can be written as the following sum of infinite second-order blocks

$$
G_{y u}(s)=\sum_{i=1}^{\infty} \frac{\alpha_{i}}{s^{2}+2 \xi_{i} \omega_{i} s+\omega_{i}^{2}}
$$

where $\alpha_{i}>0 \forall i$. Since $j\left[G_{y u}(j \omega)-G_{y u}(j \omega)^{*}\right] \geq 0 \quad \forall \quad \omega \in(0, \infty)$, the control scheme in Fig. 2 is internally stable if and only if $j\left[\hat{C}(j \omega)-\hat{C}(j \omega)^{*}\right]>$ $0 \quad \forall \omega \in(0, \infty), G_{y u}(0) \hat{C}(0)<1, G_{y u}(\infty) \hat{C}(\infty)=0$ and $\hat{C}(\infty) \geq 0$. This result demonstrates the stability of PPF and IRC control schemes [20]. How-

\footnotetext{
${ }^{1} \mathrm{~A}$ system $G(s)$ has a NIFR when $j\left[G(j \omega)-G(j \omega)^{*}\right] \geq 0$ (or $\left.>0\right)$ for all $\omega \in(0, \infty)$
} 


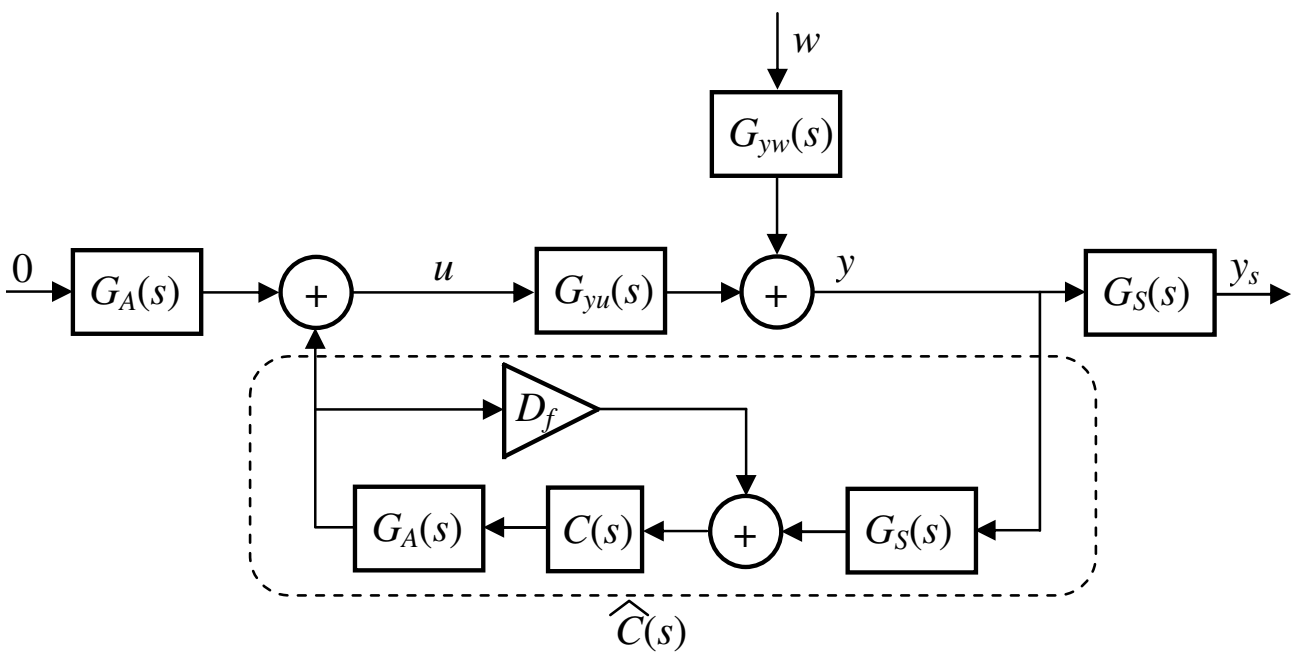

Figure 2: Equivalent representation of the general control scheme.

ever, in [20] the sensor and actuator dynamics have been neglected throughout the stability analysis. It has been seen that this simplification is not realistic in many practical cases (see for example $[22,24,23]$ ). Additionally, it has been shown that the approximation of an integral control by a band-pass filter reduces the control effort at low frequencies [18].

The inclusion of these dynamics mainly affects the phase of $\hat{C}(j \omega)$ at low-frequencies, where the value is not negative (the phase of the system not contained in $(-180,0)$ during a frequency interval). Thus, the phase of $G_{y u}(j \omega) \hat{C}(j \omega)$ could be equal to zero for $\omega \neq 0$, which makes the system unstable if $\left|G_{y u}(j \omega) \hat{C}(j \omega)\right|>1$ at this frequency. To adress this issue, this work defines a new sufficient condition that guarantees the stability of the overall system, thus extending the results proved in [19] and [20] to a controller $\hat{C}(j \omega)$ with positive values of phase in a particular (low) frequency interval. This sufficient condition states that the control scheme of Fig. 2, 
being $j\left[G_{y u}(j \omega)-G_{y u}(j \omega)^{*}\right] \geq 0$ for all $\omega \in(0, \infty)$, is internally stable if $G_{y u}(\infty) \hat{C}(\infty)=0, \hat{C}(\infty) \geq 0$ and

$$
\begin{array}{r}
j\left[\hat{C}(j \omega)-\hat{C}(j \omega)^{*}\right]>0, \omega \in\left(\omega_{c}, \infty\right), \\
\max _{\omega \in\left[0, \omega_{c}\right]}\left|G_{y u}(j \omega) \hat{C}(j \omega)\right|=\left|G_{y u}\left(j \omega_{c}\right) \hat{C}\left(j \omega_{c}\right)\right|<1
\end{array}
$$

where $\omega_{c}$ is the frequency at which $\angle \hat{C}\left(j \omega_{c}\right)=0$. Note that this necessary condition guarantees that the gain margin is greater than or equal to

$1 /\left|G_{y u}\left(j \omega_{c}\right) \hat{C}\left(j \omega_{c}\right)\right|$. Some prominent practical cases are presented here in great detail.

\subsection{Approximation of an IRC by a band-pass filter}

This approximation was presented in [18]. The control scheme of Fig. 1 is configured as follows: (a) sensor and actuator dynamics are neglected (i.e., $\left.G_{A}(s)=G_{S}(s)=1\right)$, (b) $G_{y u}(s)$ is co-located, (c) $D_{f}<0$ and (d) the TF of $C(s)$ is

$$
C(s)=\frac{\gamma s}{\left(s+p_{1}\right)\left(s+p_{1}\right)}
$$

being $\gamma>0$. Thus, the TF of $\hat{C}(s)$ is as follows

$$
\hat{C}(s)=\frac{\gamma s}{s^{2}+2 p_{1} s+p_{1}^{2}-D_{f} \gamma s}
$$

Firstly, it can be derived that the $\hat{C}(s)$ is stable if and only if $p_{1} \geq D_{f} \gamma / 2$, which is achieved since $D_{f} \gamma<0$ in order to impart damping to $G_{u y}$. Thus, the relative order of $\hat{C}(s)$ is one, $\hat{C}(\infty)=0$ and $G_{y u}(\infty) \hat{C}(\infty)=0$. There- 
fore, the stability is demonstrated if the conditions defined in Eqs. (2) and (3) are satisfied. It can be checked that $\angle \hat{C}\left(j p_{1}\right)=0^{\circ}$, which results in $\omega_{c}=p_{1}$. Then, it can be proved from the following equation

$$
j\left[\hat{C}(j \omega)-\hat{C}(j \omega)^{*}\right]=\frac{-2 \gamma \omega\left(-\omega^{2}+p_{1}^{2}\right)}{\left(-\omega^{2}+p_{1}^{2}\right)^{2}+\omega^{2}\left(2 p_{1}-D_{f} \gamma\right)^{2}}>0
$$

that condition in Eq. (2) is achieved when $\omega>\omega_{c}$.

The condition in Eq. (3) is achieved if $\left|G_{y u}(j \omega)\right|$ and $|\hat{C}(j \omega)|$ are increasing over the same interval. Firstly, the modulus of $\hat{C}(j \omega)$ can be calculated as follows:

$$
|\hat{C}(j \omega)|=\frac{\gamma}{\sqrt{\frac{1}{\omega^{2}}\left(-\omega^{2}+p_{1}^{2}\right)^{2}+\left(2 p_{1}-\gamma D_{f}\right)^{2}}},
$$

Clearly, this increases over the interval $\omega \in\left[0, \omega_{c}\right)$ if and only if the following function is decreasing

$$
f_{1}(\omega)=\frac{1}{\omega^{2}}\left(-\omega^{2}+p_{1}^{2}\right)^{2}+\left(2 p_{1}-\gamma D_{f}\right)^{2}
$$

Consequently, the first derivative of this function with respect to $\omega$ has to be less than zero over the interval $\omega \in\left[0, \omega_{c}\right)$. Then, $\mathrm{d} f_{1}(\omega) / \mathrm{d} \omega$ is calculated as follows:

$$
\frac{\mathrm{d} f_{1}(\omega)}{\mathrm{d} \omega}=2 \omega-\frac{2 p_{1}^{4}}{\omega^{3}}<0
$$


Eq. (9) is achieved if and only if

$$
\omega<p_{1}=\omega_{c}
$$

Therefore, the modulus of $\hat{C}(j \omega)$ is increasing over the interval $\omega \in\left[0, \omega_{c}\right)$.

Secondly, the modulus of $G_{y u}(j \omega)$ is increasing up to the first resonance frequency $\left(\omega_{r, 1}\right)$, which can be obtained from the experimental frequency response function (FRF). It is also can be analytically calculated as in Appendix A (see Eq. (A.6)). Thus, the control scheme is stable if

$$
p_{1}<\omega_{r, 1} \text { and }\left|G_{y u}\left(j p_{1}\right)\right|\left|\hat{C}\left(j p_{1}\right)\right|<1
$$

It should be noted that the choice of $p_{1}=0.1 \omega_{r, 1}$ was suggested in [18], guaranteeing the first part of the condition in (11).

\subsection{Inclusion of sensor dynamics in an IRC}

Although the problem of the sensor dynamics was dealt with in [22], a formal stability condition was not established. The control scheme in Fig. 1 is configured as follows: (a) the actuator dynamics is neglected (i.e., $G_{A}(s)=$ 1 ), (b) $G_{y u}(s)$ is co-located, (c) the TF of $G_{S}(s)=s /(s+p)$, (d) $D_{f}<0$ and (e) the TF of $C(s)=\gamma / s$, where $\gamma>0$. Thus, the TF of $\hat{C}(s)$ is given by:

$$
\hat{C}(s)=\frac{\gamma s}{\left(s-D_{f} \gamma\right)(s+p)}
$$

and is stable since $D_{f} \gamma<0$. Thus, the relative order of $\hat{C}(s)$ is one, $\hat{C}(\infty)=0$ and $G_{y u}(\infty) \hat{C}(\infty)=0$. Therefore, the stability is guaranteed if the conditions defined into Eqs. (2) and (3) are achieved. It can be checked that 
$\angle \hat{C}\left(j \sqrt{-D_{f} \gamma p}\right)=0^{\circ}$, which results in $\omega_{c}=\sqrt{-D_{f} \gamma p}$. Then, it can be proved from the following equation

$$
j\left[\hat{C}(j \omega)-\hat{C}(j \omega)^{*}\right]=\frac{2 \gamma \omega\left(\omega^{2}+p D_{f} \gamma\right)}{\left(-\omega^{2}+p D_{f} \gamma\right)^{2}+\omega^{2}\left(p-D_{f} \gamma\right)^{2}}>0,
$$

that condition in Eq. (2) is achieved when $\omega>\omega_{c}$.

The condition in Eq. (3) is achieved if the modulus of $G_{y u}(j \omega) \hat{C}(j \omega)$ is increasing over the interval $\omega \in\left[0, \omega_{c}\right)$. Since $\left|G_{y u}(j \omega)\right|$ is increasing for $\omega \in\left(0, \omega_{r, 1}\right)$, the condition in Eq. (3) is achieved if $|\hat{C}(j \omega)|$ is increasing for $\omega \in\left[0, \omega_{c}\right)$ and $\omega_{c}<\omega_{r, 1}$.

The modulus of $\hat{C}(j \omega)$ is given by

$$
|\hat{C}(j \omega)|=\frac{\gamma}{\sqrt{\frac{1}{\omega^{2}}\left(\omega^{2}+D_{f} \gamma p\right)^{2}+\left(p-D_{f} \gamma\right)^{2}}}
$$

which is increasing over the interval $\omega \in\left[0, \omega_{c}\right)$ if and only if the following function is decreasing

$$
f_{2}(\omega)=\frac{1}{\omega^{2}}\left(\omega^{2}+D_{f} \gamma p\right)^{2}+\left(p-D_{f} \gamma\right)^{2} .
$$

This can be demonstrated by calculating $\mathrm{d} f_{2}(\omega) / \mathrm{d} \omega$.

$$
\frac{\mathrm{d} f_{2}(\omega)}{\mathrm{d} \omega}=2 \omega-2 \frac{\left(D_{f} \gamma p\right)^{2}}{\omega^{3}}
$$

Eq. (16) can be rewritten as

$$
\omega<\sqrt{D_{f} \gamma p}=\omega_{c}
$$


Therefore, the modulus of $\hat{C}(j \omega)$ is increasing over the interval $\omega \in\left[0, \omega_{c}\right)$. Thus, the control scheme is stable if

$$
\sqrt{-D_{f} \gamma p}<\omega_{r, 1} \text { and }\left|G_{y u}\left(j \sqrt{-D_{f} \gamma p}\right)\right|\left|\hat{C}\left(j \sqrt{-D_{f} \gamma p}\right)\right|<1
$$

Note that, as mentioned in [22], the maximum value of $D_{f} \gamma$ is limited by the sensor dynamics (value of $p$ ) and the minimum gain margin required.

\subsection{Inclusion of actuator dynamics in an IRC}

The control scheme in Fig. 1 is configured as follows [23]: (a) the sensor dynamics is neglected (i.e., $G_{S}(s)=1$ ), (b) $G_{y u}(s)$ is co-located, (c) $D_{f}<$ 0 , (d) the actuator is considered as an inertial actuator with the following dynamics

$$
G_{A}(s)=\frac{K_{A} s^{2}}{s^{2}+2 \xi_{A} \omega_{A} s+\omega_{A}^{2}}
$$

where $K_{a}>0, \xi_{a}>0$ and $\omega_{i}>0$ are the system parameters and e) $C(s)$ can be defined as

$$
C(s)=\hat{G}_{A}^{-1}(s) \frac{\gamma}{s}=\frac{s^{2}+2 \xi_{A} \omega_{A} s+\omega_{A}^{2}}{K_{A}(s+p)^{2}} \frac{\gamma}{s}
$$

where $\hat{G}_{A}^{-1}(s)$ is an approximate inversion of the actuator dynamics. Thus, the transfer function of $\hat{C}(s)$ can be written as

$$
\hat{C}(s)=\frac{s \gamma}{s^{2}+\left(2 p-\gamma D_{f}\right) s+p^{2}}
$$


Note that Eq. (21) is identical to Eq. (5) if $p$ is substituted by $p_{1}$, where the control scheme is stable if Eq. (11) is satisfied.

\subsection{Inclusion of sensor dynamics in a PPF}

The control scheme in Fig. 1 is configured as follows: (a) $G_{y u}(s)$ is colocated, (b) the TF of $G_{A}(s)=1$, (c) the TF of $G_{S}(s)=s /(s+p)$, (d) $D_{f}=0$ and (e) the TF of $C(s)$ is as follows [15]

$$
C(s)=\sum_{i=1}^{N} \frac{\gamma_{i}}{s^{2}+2 \delta_{i} \tilde{\omega}_{i} s+\tilde{\omega}_{i}^{2}},
$$

where $N$ is the number of modes controlled, $\gamma_{i}>0, \delta_{i}>0$ and $\tilde{\omega}_{i}$ are tuned to impart damping to the vibration mode $i$. Thus, the TF of $\hat{C}(s)$ is given by

$$
\hat{C}(s)=\frac{s}{s+p} \sum_{i=1}^{N} \frac{\gamma_{i}}{s^{2}+2 \delta_{i} \tilde{\omega}_{i} s+\tilde{\omega}_{i}^{2}} .
$$

Although PPF control has more than one frequency where the phase of $\hat{C}(j \omega)$ is zero, the value of $\omega_{c}$ is obtained by considering $N=1$ in Eq. (26). This is because the PPF controller exhibits high-frequency roll-off characteristics and the phase at low frequencies $\left(\omega<\tilde{\omega}_{1}\right)$ is approximately equal if more vibration modes are controlled $(N>1)$. Thus, it can be deduced that

$$
\omega_{c}=\sqrt{\frac{p \tilde{\omega}_{1}^{2}}{2 \delta_{1} \tilde{\omega}_{1}+p}},
$$

and the control scheme is stable if $\left|C\left(\hat{j} \omega_{c}\right) G_{y u}\left(j \omega_{c}\right)\right|<1$. Note that $p$ should be less than $\omega_{1}$ to impart damping. 


\subsection{Inclusion of actuator dynamics in a PPF}

The control scheme in Fig. 1 is configured as follows: (a) $G_{y u}(s)$ is co-located, (b) the TF of $G_{A}(s)$ is defined as in Eq. (19), (c) the TF of $G_{S}(s)=1,(\mathrm{~d}) D_{f}=0$ and (e) the TF of $C(s)$ is defined as Eq. (22) but including the dynamics inversion used into Eq. (20) given by

$$
C(s)=\frac{s^{2}+2 \xi_{A} \omega_{A} s+\omega_{A}^{2}}{K_{A}(s+p)^{2}} \sum_{i=1}^{N} \frac{\gamma_{i}}{s^{2}+2 \delta_{i} \tilde{\omega}_{i} s+\tilde{\omega}_{i}^{2}} .
$$

Thus, the TF of $\hat{C}(s)$ can be written as

$$
\hat{C}(s)=\frac{s^{2}}{(s+p)^{2}} \sum_{i=1}^{N} \frac{\gamma_{i}}{s^{2}+2 \delta_{i} \tilde{\omega}_{i} s+\tilde{\omega}_{i}^{2}} .
$$

Applying the same conditions and performing the same analysis as detailed in the previous section, the control scheme is stable if $\left|\hat{C}\left(j \omega_{c}\right) G_{y u}\left(j \omega_{c}\right)\right|<1$, where $\omega_{c}$ is given by

$$
\omega_{c}=\sqrt{\frac{p \tilde{\omega}_{1}^{2}+\delta_{1} \tilde{\omega}_{1} p^{2}}{\delta_{1} \tilde{\omega}_{1}+p}} .
$$

\section{Example}

This example shows how to tackle problems introduced by the sensor dynamics during an IRC implementation. In addition, this section also explains why a band-pass filter is better than pure integral action for $C(s)$ in this particular case. The transfer functions of the cantilever beam instrumented with piezoelectric patches as employed in [22] have been utilized to obtain simulation results for both controllers options. 
The design methodology for IRC is briefly explained. According to [18], and neglecting the actuator and sensor dynamics $\left(G_{A}(s)=G_{S}(s)=1\right)$, the loop dynamics used to tune IRC parameters in [18] is given by

$$
G_{L, 1}(s)=\left(G_{y u}(s)+D_{f}\right) C(s)
$$

The design methodology proposed in [18], which is shown in Fig. 3, can be itemized as: (a) determine the maximum value of $D_{f}$ to guarantee the stability of the controlled system w.r.t. $D_{f}<-G_{y u}(0)$, by adding a zero at a frequency lower than the first resonant mode of the system (Fig. 3b), (b) place this zero as a far away from the first resonance pole, thereby increasing the maximum imparted damping, and (c) design $C(s)$, given by $C(s)=\gamma / s$ as shown in Fig. 3.c, to impart damping to the system. A band-filter can be used instead, (Eq. (4)), where $p_{1}<<\omega_{r, 1}$ (for example, $p_{1}=0.1 \omega_{r 1}$ ).

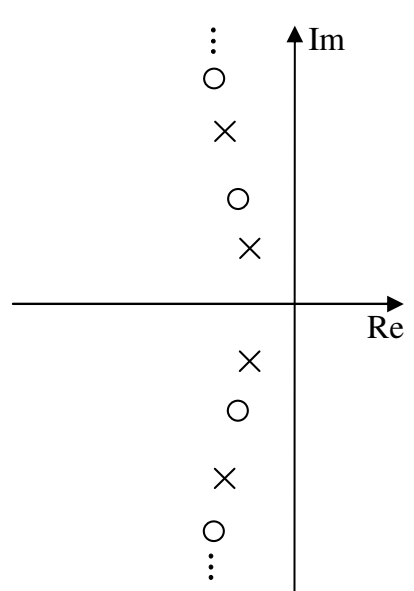

(a)

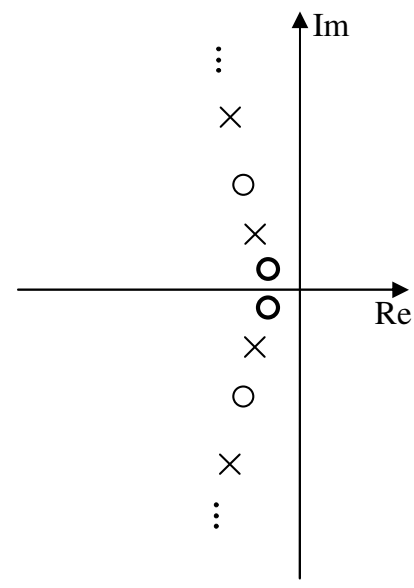

(b)

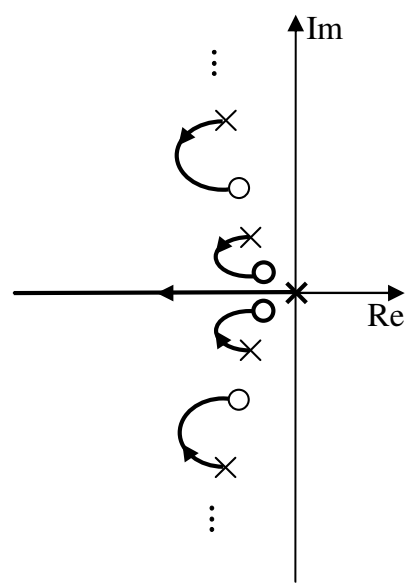

(c)

Figure 3: Design method proposed in [18]. (a) Pole-Zero diagram of $G_{y u}(s)$. (b) Pole-Zero diagram of $G_{y u}(s)+D_{f}$. (c) Root locus of $\left(G_{y u}(s)+D_{f}\right)(\gamma / s)$. 
However, if the sensor dynamics cannot be neglected, the design proposed in [18] is not adequate, since $G_{S}(s)$ will affect the imparted damping possible. In this case, [22] proposed to use the characteristic equation given by

$$
G_{L, 2}(s)=\frac{C(s)}{1-C(s) D_{f}} G_{S}(s) G_{y u}(s)
$$

where $D_{f}$ and $\gamma$ were chosen from the root locus given in Fig. 4. If the sensor dynamics is not considered $\left(G_{S}(s)=1\right)$, the damping imparted by the controller depends on the position of the real pole $\gamma D_{f}$ and the gain $-1 / D_{f}$ (see Fig. 4.a). In addition, the stability condition is $-G_{y u}(0) / D_{f}<1$, which is similar to the condition defined in [18]. If $G_{S}(s)=s /(s+p)$, the choice of the real pole $\gamma D_{f}$ and the gain $-1 / D_{f}$ depends on $-p$ (see Fig. 4.b). In addition, the stability condition is now defined by Eq. (18). It should be remarked that, as shown in [22], the maximum damping is limited by the position of $-p$. Thus, the maximum imparted damping is less when the value of $-p$ is bigger.

An alternative design can be proposed, where $C(s)$ is defined as

$$
C(s)=\frac{\gamma\left(s+z_{1}\right)}{\left(s+p_{1}\right)\left(s+p_{1}\right)}
$$

Thus, the transfer function of $\hat{C}(s)$ if the sensor dynamics is considered is

$$
\hat{C}(s)=\frac{\gamma\left(s+z_{1}\right)}{s^{2}+2 p_{1} s+p_{1}^{2}-D_{f} \gamma s} \frac{s}{s+p} .
$$

Then, if $z_{1}=p_{1}$, a controller structurally similar to Eq. (5) can be derived and the design methodology defined in [18], can be used when $p_{1}<<\omega_{r, 1}$. 


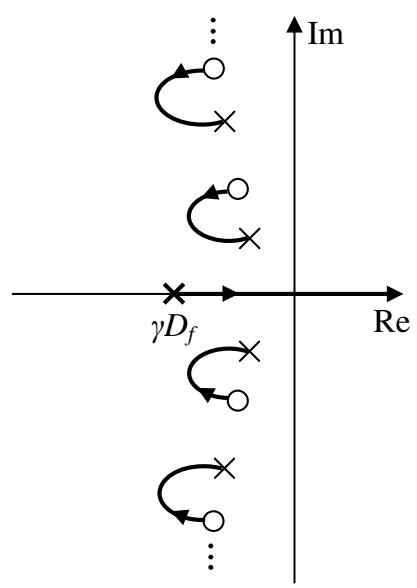

(a)

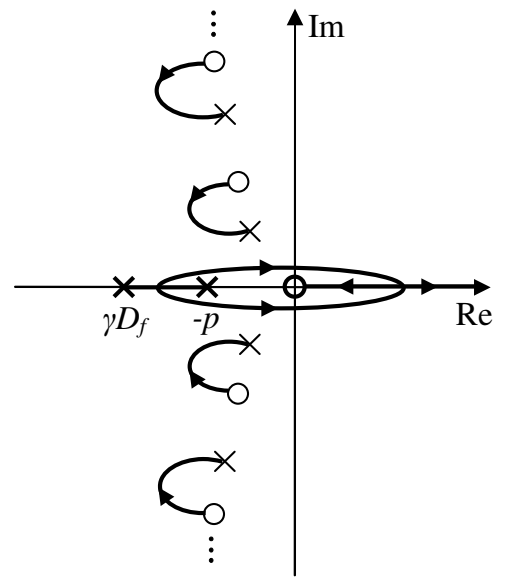

(b)

Figure 4: Design method proposed in [22]. (a) Pole-Zero diagram when $C(s)=\gamma / s$. (b) Pole-Zero diagram when $C(s)=\gamma / s$ and $G_{S}(s)=s /(s+p)$.

In addition, the stability condition can now be defined by Eq. (11).

\subsection{Simulation results}

The identified model of the cantilever beam used in [22] (see Fig. 5) is used to illustrate the two possible designs when the sensor dynamics is considered. The state-space model is as follows 


$$
\begin{aligned}
& \dot{\mathbf{x}}=\left[\begin{array}{cccccc}
0 & 1 & 0 & 0 & 0 & 0 \\
-2799 & -0.8 & 0 & 0 & 0 & 0 \\
0 & 0 & 0 & 1 & 0 & 0 \\
0 & 0 & -1.024 \cdot 10^{5} & -4 & 0 & 0 \\
0 & 0 & 0 & 0 & 0 & 1 \\
0 & 0 & 0 & 0 & -7.868 \cdot 10^{5} & -11.09
\end{array}\right] \mathbf{x}+ \\
& {\left[\begin{array}{cc}
0 & 0 \\
1 & 0.490 \\
0 & 0 \\
1 & -1.148 \\
0 & 0 \\
1 & -1.679
\end{array}\right]\left[\begin{array}{ll}
w & u
\end{array}\right]} \\
& {\left[\begin{array}{l}
y \\
z
\end{array}\right]=\left[\begin{array}{cccccc}
-0.205 & 0 & 0.789 & 0 & -1.222 & 0 \\
13.23 & 0 & -359.5 & 0 & -1089 & 0
\end{array}\right] \mathbf{x}+} \\
& {\left[\begin{array}{cc}
4.411 \cdot 10^{-7} & 1.261 \cdot 10^{-6} \\
-1.114 \cdot 10^{-3} & 4.477 \cdot 10^{-2}
\end{array}\right]\left[\begin{array}{ll}
w & u
\end{array}\right]}
\end{aligned}
$$

and the sensor dynamics was identified as

$$
G_{S}(s)=\frac{s}{s+5} .
$$

The transfer function $G_{y u}(s)$ derived from the model of Eq. (32) is given by 


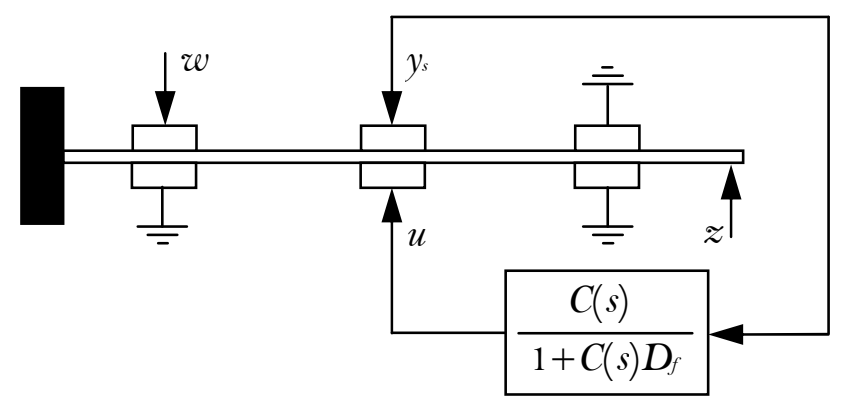

Figure 5: System model, where $u$ is the input and $y$ is the output of the co-located patch, $w$ is the disturbance patch and $z$ is the tip velocity.

$$
\begin{array}{r}
G_{y u}(s)=0.045 \cdot \frac{\left(s^{2}+0.80 s+2925\right)}{\left(s^{2}+0.80 s+2799\right)} \\
\frac{\left(s^{2}+4.01 s+1.11 \cdot 10^{5}\right)}{\left(s^{2}+4.00 s+1.02 \cdot 10^{5}\right)} \cdot \frac{\left(s^{2}+11.08 s+8.28 \cdot 10^{5}\right)}{\left(s^{2}+11.09 s+7.87 \cdot 10^{5}\right)}
\end{array}
$$

where $\omega_{r, 1}=52.91 \mathrm{rad} / \mathrm{s}$, and is used to design the IRC as described in Sections 3.1 and 3.2 .

First, the parameters $D_{f}$ and $\gamma$ are chosen according to [18] for the following cases: (Case a) $G_{y u}(s)$ without considering $G_{S}(s)$ and $C(s)=\gamma / s$, (Case b) $G_{y u}(s)$ together with $G_{S}(s)$ and $C(s)=\gamma / s$ and (Case c) $G_{y u}(s)$ together with $G_{S}(s)$ and $C(s)$ as defined in Eq. (31) for $z_{1}=p=5$ and $p_{1}=5<0.1 \omega_{r, 1}$.

If $G_{S}(s)$ is not considered, $D_{f}$ must be less than $-G_{y u}(0)=-0.053$. The following five values of $D_{f}$ are identified $D_{f}=\left\{-G_{y u}(0) / 0.5,-G_{y u}(0) / 0.6\right.$, $\left.-G_{y u}(0) / 0.7,-G_{y u}(0) / 0.8,-G_{y u}(0) / 0.9\right\}$. The maximum damping ratios obtained for each value of $D_{f}$ and its corresponding value of $\gamma$ are summarized 
in Table 1. In addition, these damping ratios are also calculated for (Case b) and (Case c) by using the same values of $D_{f}$ and $\gamma$. It can be deduced from Table 1 that: (a) the damping ratios are increased when $G_{S}(s)$ is not considered as long as $D_{f}$ is increased (until the limit of $D_{f}=-G_{y u}(0)$ ), (b) the damping ratios are also increased in the same way when $G_{S}(s)$ is considered together with $C(s)$ defined as Eq. (31) for $z_{1}=p=5$ and (c) the design criterion of [18] is not useful when $G_{S}(s)$ is considered together with $C(s)=\gamma / s$ since $\xi_{1}$ increases only up to a certain value of $D_{f}$.

Secondly, the design used in [22] when $C(s)=\gamma / s$ is considered. Here, the pole $D_{f} \gamma$ was placed by considering the dynamics of $G_{S}(s)$. Thus, the maximum imparted damping can be increased by moving away $D_{f} \gamma$ from the origin up to a certain value. Then, the minimum value for $D_{f} \gamma$ was selected as -226.2 and the controller gain was $1 / D_{f}=-18$ [22]. If the stability condition in Eq. (18) is used, it results as follows

$$
\begin{array}{r}
\sqrt{-D_{f} \gamma p}=33.63<\omega_{r, 1}=52.91 \text { and } \\
\left|G_{y u}\left(j \sqrt{-D_{f} \gamma p}\right)\right|\left|\hat{C}\left(j \sqrt{-D_{f} \gamma p}\right)\right|=0.9768<1
\end{array}
$$

showing that the control scheme is stable. In addition, the damping ratios are $\xi_{1}=0.101, \xi_{2}=0.0321$ and $\xi_{3}=0.0116$, which show that the value of $\xi_{1}$ is close to $D_{f}=-G_{y u}(0) / 0.9$ and $\gamma=6.73 \cdot 10^{3}$ without considering the $G_{S}(s)$. However, the values for $\xi_{2}$ and $\xi_{3}$ are smaller. If the band-pass filter is utilized, the same damping can be achieved by the following variations to the original design: (a) increasing $D_{f}$ and $\gamma$, which reduces the stability margins of the controlled system and/or (b) reducing the value of $p_{1}$, which 
Table 1: Damping ratios when the design criterion of [18] is used.

\begin{tabular}{|c|c|c|c|}
\hline \multicolumn{4}{|c|}{$D_{f}=-G_{y u}(0) / 0.5$ and $\gamma=1.18 \cdot 10^{3}$} \\
\hline & $\xi_{1}$ & $\xi_{2}$ & $\xi_{3}$ \\
\hline Case a & 0.0185 & 0.0134 & 0.0078 \\
\hline Case b & 0.0166 & 0.0134 & 0.0078 \\
\hline Case c & 0.0166 & 0.0133 & 0.0078 \\
\hline \multicolumn{4}{|c|}{$D_{f}=-G_{y u}(0) / 0.6$ and $\gamma=1.71 \cdot 10^{3}$} \\
\hline & $\xi_{1}$ & $\xi_{2}$ & $\xi_{3}$ \\
\hline Case a & 0.0240 & 0.0167 & 0.0085 \\
\hline Case b & 0.0208 & 0.0167 & 0.0085 \\
\hline Case c & 0.0211 & 0.0165 & 0.0085 \\
\hline \multicolumn{4}{|c|}{$D_{f}=-G_{y u}(0) / 0.7$ and $\gamma=2.46 \cdot 10^{3}$} \\
\hline & $\xi_{1}$ & $\xi_{2}$ & $\xi_{3}$ \\
\hline Case a & 0.0333 & 0.0213 & 0.0095 \\
\hline Case b & 0.0276 & 0.0214 & 0.0095 \\
\hline Case $c$ & 0.0287 & 0.0211 & 0.0095 \\
\hline \multicolumn{4}{|c|}{$D_{f}=-G_{y u}(0) / 0.8$ and $\gamma=3.89 \cdot 10^{3}$} \\
\hline & $\xi_{1}$ & $\xi_{2}$ & $\xi_{3}$ \\
\hline Case a & 0.0518 & 0.0301 & 0.0113 \\
\hline Case b & 0.0366 & 0.0303 & 0.0113 \\
\hline Case c & 0.0476 & 0.0299 & 0.0113 \\
\hline \multicolumn{4}{|c|}{$D_{f}=-G_{y u}(0) / 0.9$ and $\gamma=6.73 \cdot 10^{3}$} \\
\hline & $\xi_{1}$ & $\xi_{2}$ & $\xi_{3}$ \\
\hline & 0.1070 & 0.0480 & 0.0150 \\
\hline Case b & 0.0117 & 0.0486 & 0.0150 \\
\hline Case c & 0.0871 & 0.0476 & 0.0150 \\
\hline
\end{tabular}

reduces the gain attenuation at low frequencies. For example, if $p_{1}=3$, $D_{f}=-G_{y u}(0) / 0.91$ and $\gamma=6.8 \cdot 10^{3}$, the damping ratios are $\xi_{1}=0.103$, $\xi_{2}=0.0484$ and $\xi_{3}=0.0151$. Applying the stability condition in Eq. (11), results in

$$
\begin{array}{r}
p_{1}=3<\omega_{r, 1}=52.91 \text { and } \\
\left|G_{y u}\left(j \sqrt{-D_{f} \gamma p}\right)\right|\left|\hat{C}\left(j \sqrt{-D_{f} \gamma p}\right)\right|=0.9483<1
\end{array}
$$




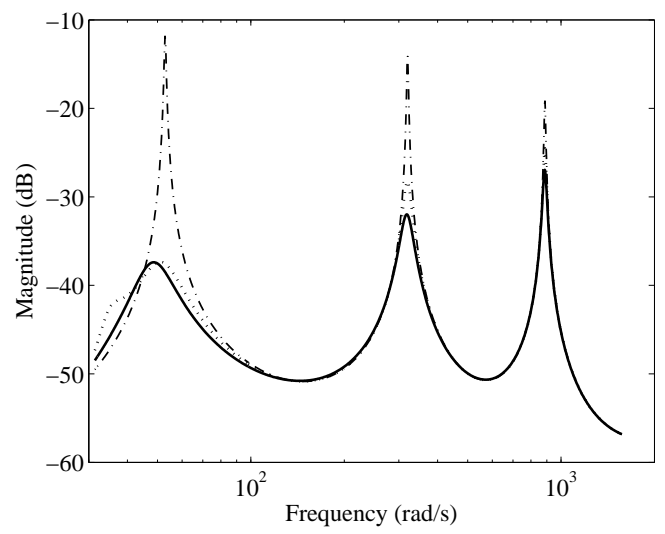

Figure 6: FRF of $G_{z w}$ for the first three vibration modes. (- - -) open-control, (-) closed-loop with $C(s)$ of Eq. (30) for $p_{1}=3, D_{f}=-G_{y u}(0) / 0.91$ and $\gamma=6.8 \cdot 10^{3}$ and $(--)$ closed-loop with $C(s)=\gamma / s$ for $D_{f} \gamma=-226.2$ and $1 / D_{f}=-18$.

which shows that the control scheme is stable. Simulation results were carried out as a check and are summarized in Fig. 6. It can be seen that the amplitude of the second and third vibration mode for $C(s)=\gamma s /\left(s+p_{1}\right)^{2}$ are, respectively, $3.7 \mathrm{~dB}$ and $2.5 \mathrm{~dB}$ less than for $C(s)=\gamma / s$. Thus, this example shows that the band-pass filter mitigates the problem associated to the sensor dynamics.

\section{Conclusion}

This work has shown that stability theory based on Negative-Imaginary Systems can be extended for control schemes that include low-frequency restrictions. Simple and analytical stability conditions have been defined for a group of low-frequency restrictions that commonly appear in practice. In addition, the work includes a comparison between two different controllers, showing how to apply the formulated stability conditions to tune the con- 
trollers and to guarantee the stability of the overall system.

\section{Appendix A. Resonance frequency of $G_{y u}$}

The transfer function in Eq. (1) can be rewritten as follows

$$
G_{y u}(s)=G_{y u}(0) \prod_{i=1}^{\infty} G_{y u}^{i}(s)=G_{y u}(0) \prod_{i=1}^{\infty} \frac{s^{2} /\left(\omega_{z, i}^{2}\right)+2 \xi_{z, i} s /\left(\omega_{z, i}\right)+1}{s^{2} /\left(\omega_{i}^{2}\right)+2 \xi_{i} s /\left(\omega_{i}\right)+1},
$$

where $\omega_{i}<\omega_{z, i} \forall i$ due to the pole-zero interlacing property of co-located systems [25]. Thus, it can be demonstrated that $\left|G_{y u}(j \omega)\right|$ is increasing if every block in Eq. (A.1) is increasing over the interval $\omega \in\left(0, \omega_{c}\right)$. The modulus of $i^{\text {th }}$ block $\left(G_{y u}^{i}(s)\right)$ is as follows:

$$
\left|G_{y u}^{i}(j \omega)\right|=\sqrt{\frac{\left(1-\omega^{2} /\left(\omega_{z, i}^{2}\right)\right)^{2}+4 \omega^{2} \xi_{z, i}^{2} /\left(\omega_{z, i}^{2}\right)}{\left(1-\omega^{2} /\left(\omega_{i}^{2}\right)\right)^{2}+4 \omega^{2} \xi_{i}^{2} /\left(\omega_{i}^{2}\right)}} .
$$

After deriving Eq. (A.2) and simplifying further, the following increasing condition can be defined:

$$
\begin{aligned}
& {\left[-4 \omega\left(1-\omega^{2} / \omega_{z, i}^{2}\right) / \omega_{z, i}^{2}+8 \xi_{z, i}^{2} \omega / \omega_{z, i}^{2}\right]\left[\left(1-\omega^{2} / \omega_{i}^{2}\right)^{2}+4 \omega^{2} \xi_{i}^{2} / \omega_{i}^{2}\right]-} \\
& {\left[\left(1-\omega^{2} / \omega_{z, i}^{2}\right)^{2}+4 \omega^{2} \xi_{z, i}^{2} / \omega_{z, i}^{2}\right]\left[-4 \omega\left(1-\omega^{2} / \omega_{i}^{2}\right) / \omega_{i}^{2}+8 \xi_{i}^{2} \omega / \omega_{i}^{2}\right]>0}
\end{aligned}
$$

If Eq. (A.3) is simplified, it results in

$$
\omega^{4} \Delta_{1}+\omega^{2} \Delta_{2}+\Delta_{3}>0
$$


where $\Delta_{1}, \Delta_{2}$ and $\Delta_{3}$ are given by:

$$
\begin{array}{r}
\Delta_{1}=\omega_{z, i}^{2}\left(1-2 \xi_{z, i}^{2}\right)-\omega_{i}^{2}\left(1-2 \xi_{i}^{2}\right) \\
\Delta_{2}=\omega_{i}^{4}-\omega_{z, i}^{4} \\
\Delta_{3}=\omega_{i}^{2} \omega_{z, i}^{2}\left[\omega_{z, i}^{2}\left(1-2 \xi_{i}^{2}\right)-\omega_{i}^{2}\left(1-2 \xi_{z, i}^{2}\right)\right] .
\end{array}
$$

Thus, the resonance frequency of each $G_{y u}^{i}(s)$ can be calculated from the expression,

$$
\omega_{r, i}=\sqrt{\frac{-\Delta_{2}-\sqrt{\Delta_{2}^{2}-4 \Delta_{1} \Delta_{3}}}{2 \Delta_{1}}},
$$

where each $\left|G_{y u}^{i}(j \omega)\right|$ is increasing up to $\omega_{r, i}$.

\section{References}

[1] R. H. Canon, E. Schmitz, Initial experiments on the end-point control of a flexible robot, International Journal on Robotics Research 3 (3) (1984) $62-75$.

[2] S. S. Han, S. B. Choi, H. H. Kim, Postition control of a flexible gantry robot arm using smart material actuators, Journal of Robotics Systems 16 (10) (1999) 581-595.

[3] S. Wu, T. L. Turner, S. A. Rizzi, Piezoelectric shunt vibration damping of F-15 panel under high-acoustic excitation, in: Proceddings of SPIE. Smart Structures and Materials: Damping and Isolation, 2000, pp. 276287. 
[4] M. Tokhi, Z. Mohamed, M. Shaheed, Dynamic characterisation of a flexible manipulator system, Robotica 19 (2001) 571-580.

[5] L. Vaillon, C. Philippe, Passive and active microvibration control for very high pointing accuracy space systems, Smart Materials and Structures 8 (1999) 719-728.

[6] S. Salapaka, A. Sebastian, J. P. Clevland, M. Salapaka, Design identification and control of a fast nanopositioning device, in: Proccedings of American Control Conference, 2002, pp. 1966-1971.

[7] M. Benosman, G. Vey, Control of flexible manipulators: A survey, Robotica 22 (2004) 533-545.

[8] Y. Hu, A. Ng, Active robust vibration control of flexible structures, Journal of Sound and Vibration 288 (1-2) (2005) 43-46.

[9] M. J. Balas, Feedback control of flexible system, IEEE Transactions on Automatic Control Engineering Practice 23 (1978) 673-679.

[10] M. Zilletti, S. J. Elliott, P. Gardonio, Self-tuning control systems of decentralised velocity feedback, Journal of Sound and Vibration 329 (14) (2010) 2738-2750.

[11] S. O. R. Moheimani, A. J. Fleming, Piezoelectric transducers for vibration control and damping, Springer Verlag, London Limited, 2006.

[12] S. O. R. Moheimani, D. Halim, Spatial resonant control of flexible structures - application to a piezoelectric laminated beam, IEEE Transactions on Control Systems Technology 9 (1) (2001) 37-53. 
[13] H. R. Pota, S. O. R. Moheimani, Resonant controllers for smart structures, Smart Materials and Structures 11 (2002) 1-8.

[14] S. O. R. Moheimani, B. J. G. Vautier, Resonant control of structural vibration using charge-driven piezoelectric actuators, IEEE Transactions on Control System Technology 13 (6) (2005) 1021-1035.

[15] J. L. Fanson, T. K. Caughey, Positive position feedback-control for large space structures, AIAA Journal 28 (4) (1990) 717-724.

[16] S. O. R. Moheimani, B. J. G. Vautier, B. Bhikkaji, Experimental implementation of extended multi-variable PPF control on an active structure, IEEE Transactions on Control Systems Technology 14 (3) (2006) 443-445.

[17] Z. C. Qiu, X. M. Zhanga, H. X. Wub, H. H. Zhangb, Optimal placement and active vibration control for piezoelectric smart flexible cantilever plate, Journal of Sound and Vibration 301 (3-5) (2007) 521-543.

[18] S. S. Aphale, A. J. Fleming, S. O. R. Moheimani, Integral resonant control of collocated smart structures, Smart Materials and Structures 16 (2007) 439-446.

[19] A. Lanzon, I. R. Petersen, Stability robustness of a feedback interconnection of systems with negative imaginary frequency response, IEEE Transactions of Automatic Control 53 (4) (2008) 1042-1046.

[20] I. R. Petersen, A. Lanzon, Feedback control of negative-imaginary systems, IEEE Control Systems Magazine 30 (5) (2010) 54-72. 
[21] S. Patra, A. Lanzon, Stability analysis of interconnected systems with mixed negative- imaginary and small-gain properties, IEEE Transactions on Automatic Control 56 (6) (2011) 1395-1400.

[22] E. Pereira, S. O. R. Moheimani, S. S. Aphale, Analog implementation of the integral resonant control scheme, Smart Materials and Structures 16 (6) (2008) 1-6.

[23] I. M. Díaz, E. Pereira, P. Reynolds, Integral resonant control scheme for cancelling human-induced vibrations in light-weight pedestrian structures, Structural Control and Health Monitoring 19 (2012) 55-69.

[24] E. Pereira, S. S. Aphale, V. Feliu, S. O. R. Moheimani, Integral resonant control for vibration damping and precise tip-positioning of a single-link flexible manipulator, IEEE/ASME Transactions on Mechatronics 16 (2) (2011) 232-240.

[25] O. J. M. Smith, Feedback Control Systems, New York: McGraw-Hill Book Co., Inc, 1958. 


\section{List of Tables}

1 Damping ratios when the design criterion of [18] is used. . . . 20 


\section{List of Figures}

1 Positive-position feedback. General control scheme. . . . . . . 5

2 Equivalent representation of the general control scheme. . . . . 6

3 Design method proposed in [18]. (a) Pole-Zero diagram of $G_{y u}(s)$. (b) Pole-Zero diagram of $G_{y u}(s)+D_{f}$. (c) Root locus of $\left(G_{y u}(s)+D_{f}\right)(\gamma / s) \ldots \ldots \ldots \ldots \ldots$

4 Design method proposed in [22]. (a) Pole-Zero diagram when $C(s)=\gamma / s$. (b) Pole-Zero diagram when $C(s)=\gamma / s$ and $G_{S}(s)=s /(s+p) \ldots \ldots \ldots \ldots \ldots \ldots$

5 System model, where $u$ is the input and $y$ is the output of the co-located patch, $w$ is the disturbance patch and $z$ is the tip velocity. . . . . . . . . . . . . . . . 18

6 FRF of $G_{z w}$ for the first three vibration modes. $(-\cdot-)$ opencontrol, (-) closed-loop with $C(s)$ of Eq. (30) for $p_{1}=3$, $D_{f}=-G_{y u}(0) / 0.91$ and $\gamma=6.8 \cdot 10^{3}$ and (--) closed-loop with $C(s)=\gamma / s$ for $D_{f} \gamma=-226.2$ and $1 / D_{f}=-18 \ldots . .21$ 